\title{
Constraints on autoshaping in the squirrel monkey: Stimulus and response factors
}

\author{
ELIAS SCHWAM and ELKAN GAMZU \\ Pharmacology Department, Research Division, Hoffmann-La Roche, Inc. \\ Nutley, New Jersey 67110
}

\begin{abstract}
Lever responding in four of five naive squirrel monkeys was autoshaped when a visual stimulus was paired with food presentations. Despite topographical similarity between the autoshaped response and the grasping of food, neither lever responding nor measurable approach behavior was maintained when the occurrence of lever responding precluded the delivery of food. In the second experiment, in which an auditory stimulus signaled food presentations, autoshaping occurred when the manipulandum was constantly illuminated. The rate of acquisition was slower than when a visual stimulus signaled food, although responding was comparably eliminated in the negative response-dependency procedure. Variability in the patterns of behavior leading to consumption in the squirrel monkey may account for the differences between the behavior of this species and pigeons when exposed to these procedures.
\end{abstract}

The results of autoshaping procedures in the squirrel monkey, in which key illumination is paired with presentation of food, contrast sharply with the reported findings in pigeons. Acquisition of the autoshaped response requires only 6-119 trials for pigeons (Brown \& Jenkins, 1968) vs. 500-600 trials for squirrel monkeys (Gamzu \& Schwam, 1974). Secondly, responding is maintained in pigeons even when responses preclude the delivery of food (negative automaintenance, Williams \& Williams, 1969) while responding is virtually eliminated by this procedure in squirrel monkeys (negative response-dependency, Gamzu \& Schwam, 1974). Thirdly, while a response-independent fixed-trial procedure can maintain high rates of responding in the pigeon (Brown \& Jenkins, 1968), it results in a marked reduction of responding in squirrel monkeys (Gamzu $\hat{\&}$ Schwam, 1974). Finally, topographical similarity between the autoshaped and consummatory responses (Jenkins \& Moore, 1973) has been demonstrated in the pigeon. With squirrel monkeys, however, both the autoshaped keypress response and the patterns of behavior leading to consumption are variable (Gamzu \& Schwam, 1974).

Gamzu and Schwam (1974) suggested that the source of these differences may well lie in the nature of the topographies of autoshaped and consummatory responses. Indeed, among the predominant findings in the autoshaping literature is the similarity between the learned response and the unconditioned response evoked by the reinforcer (Jenkins \& Moore, 1973; Peterson, Ackil, Frommer, \& Hearst, 1972; Squier, 1969). It has

Experiment I was presented at the 44th Annual Meeting of the Eastern Psy chological Associaton, Washington, D. C., May 4, 1973. The authors are particularly indebted to Barbara Salsitz, Jerry Sepinwall, and Larry Byrd for their comments and suggestions and Joseph Sia for his technical assistance. We also thank Diana Cantrella and Linda Volpe for their help in preparation of this manuscript. Reprints may be obtained from Elkan Gamzu, Department of Pharamacology, Hoffmann-La Roche Inc., Nutley, New Jersey 07110 . been suggested that this similarity represents, or is mediated by, the operation of Pavlovian-like stimulus-reinforcer associations (Gamzu \& Schwam, 1974; Gamzu \& Williams, 1971, 1973; Jenkins \& Moore, 1973; Moore, 1973). As has been pointed out (Gamzu and Schwam, 1974; Schwartz \& Williams, 1972a, 1972b; Williams \& Williams, 1969), it is the negative automaintenance (or negative response dependency) procedure which represents the most potent demonstration of stimulus-reinforcer control.

On the assumption that both topographical similarity and negative automaintenance are mediated by the same Pavlovian-like mechanism, would the demonstration of topographical similarity in the squirrel monkey, like the pigeon, also be accompanied by maintenance of responding on the negative response-dependency procedure? To answer this, the first experiment employed an omnidirectional lever manipulandum which educes a grasping response similar to that of reaching for a food pellet.

\section{EXPERIMENT I}

\section{Methods}

Subjects. Subjects were five naive adult male squirrel monkeys (Saimiri sciureus) maintained at free feeding weights. They were $22 \mathrm{~h}$ food deprived at the beginning of each session.

Apparatus. Subjects were studied in BRS-Foringer experimental chamber with internal measurements of $17 \times 17 \times 14$ in. $(43 \times 43 \times 36 \mathrm{~cm})$. On one wall, painted flat black, a $3 / 4$ in. $(1.9 \mathrm{~cm})$ long omnidirectional lever was positioned $10 \mathrm{in} .(25.4 \mathrm{~cm})$ above the grid floor in a recessed housing and a small 1.1-W light was mounted over the lever.

About 6 in. $(15.2 \mathrm{~cm})$ below the lever a small wire mesh foodcup extended into the chamber. Delivery of $120-\mathrm{mg}$ food pellets (Riddle, Rednick, Catania \& Tucker, 1966) was accompanied by a slight click of the feeder mechanism. Three attenuated 2-W white houselights, positioned above the experimental walls and low amplitude masking noise were on throughout the session. The illumination from the house lights was deflected toward the ceiling of the chamber. 
Sessions were controlled by standard BRS-Foringer circuitry and monitored by closed circuit television.

Procedure. Following magazine training all monkeys were exposed to the autoshaping procedure. This procedure consisted of pairings of illumination of the light above the lever and presentation of food. If during the trial, a lever response was made, the trial ended immediately with the delivery of a pellet. If the monkey failed to respond, the pellet was delivered at the end of $10 \mathrm{sec}$. The monkeys were exposed to this procedure until they were responding on $90 \%$ of the trials for about 5 consecutive days.

Monkeys 323 and 302 were then exposed to the negative response-dependency procedure. Here the pellet was delivered at the end of the 10 -sec trial as in autoshaping. If a lever response occurred, however, the trial terminated immediately without the delivery of the pellet.

Subjects were studied 5 days a week. A session consisted of 50 10 -sec trials during which the light above the lever was illuminated. The mean intertrial interval was $40 \mathrm{sec}$ with an approximately geometric distribution.

\section{Results}

Autoshaping. In four of the five monkeys the lever response was successfully autoshaped. As shown in acquisition curves in Figure 1, Monkeys 323, 249, 302, and 332 made their first lever response during Sessions $4,8,2$, and 3, respectively. Monkey 285 , whose data are not shown, failed to make a single lever response over the course of 31 sessions.

For Monkeys 323 and 249 acquisition of lever responding took from 15-20 sessions while Monkey 302 reached $90 \%$ responding after only eight sessions. Although Monkey 332 reached a maximum of only 40\%-50\% responding over the full 50 trial session, analysis of the first half of each session indicate that responding during this period reached $70 \%-80 \%$ by Session 27 . Both an usually high level of motor activity and atypical failure to consume pellets were concomitant with the reduction of lever responding by this animal during the second half of most sessions. For these four monkeys there was a relatively large number of intertrial interval responses at the beginning of acquisition, but with the exception of Monkey 323, stimulus control was evident once they were responding consistently.

Negative response-dependency. This procedure resulted in a rapid reduction of lever responding such that lever responding was virtually eliminated in both monkeys after 5-6 sessions.

\section{Discussion}

The use of the lever manipulandum, while educing a grasping response more similar in topography to that of grasping a food pellet, did not lead to more rapid acquisition of the autoshaped response than was obtained with a keypress manipulandum (Gamzu \& Schwam, 1974). Similarly, the lever response, like the keypress, was not maintained when its occurrence precluded the delivery of food.

While the monkeys in this study, as well as in the previous study (Gamzu \& Schwam, 1974) were consistently observed to make forehand grasping responses in obtainining food peilets, a

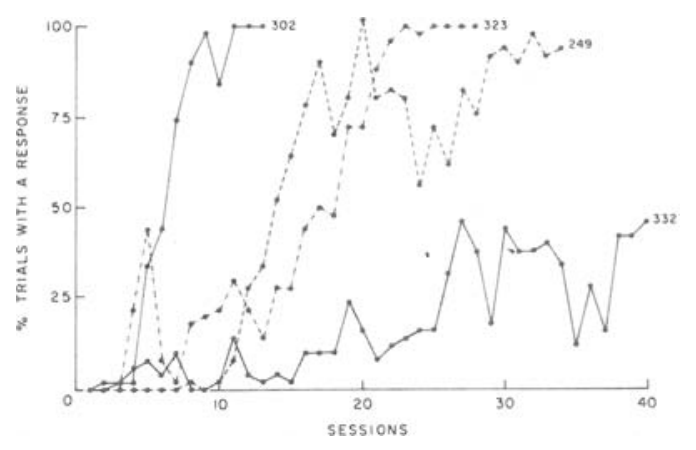

Figure 1. Acquisition of leverpressing by autoshaping in four squirrel monkeys. Percent of daily trials to which there was a response.

host of behaviors are possible between this response and the more final consummatory response of ingestion. Indeed, sometimes a grasped food pellet is brought directly to the monkey's mouth; other times it is rubbed either between the hands, the hands and the tail, or the hands and a wall of the experimental chamber. Contrast this with the "brevity and relative stereotype of the feeding response components" of the pigeon (Zeigler \& Welch, Note 1 ; see also Zeigler, 1974). In the monkey, the greater variety of possible behaviors between grasping and ingestion suggests that these behaviors should be susceptible to inhibition by behaviorally produced outcomes and relatively insensitive to direct control by stimulus-reinforcer pairings.

While the grasping response is not susceptible to stimulus-reinforcer control, stimulus-reinforcer relationships are nevertheless necessary for the acquisition of autoshaped responding in the squirrel monkey (Gamzu \& Schwam, 1974). What component of behavior is sensitive to associative control, however, has not yet been determined. It is entirely possible that some component of orientation of approach, e.g., head or eye movements, might have been sensitive to stimulus reinforcer control. Indeed, such a component appeared to be present in the approach behavior of Monkey 302. On almost every trial of the negative-response dependency procedure this monkey would rise up with one forelimb partially extended, while facing the lever. Even this response was eliminated, however, when its occurrence immediately terminated the trial and precluded the delivery of food.

While the variability in the pattern of behavior leading to consumption in the monkey may account for these findings, it is also possible that rapidity of acquisition may be influenced by the nature of the signaling stimulus. Indeed, as Wasserman (1973) has demonstrated, autoshaping of the pigeon's keypeck depends upon a highly localized visual cuing stimulus. On the other hand, when auditory stimuli signal food presentations, pecking at the sound source seldom occurs (Bilbrey \& Winokur, 1973; Farthing, 1971). The second experiment reported here is concerned with the dimension of the signaling stimulus.

\section{EXPERIMENT II}

Control of keypress responding in squirrel monkeys by localization of auditory stimuli has been reported by Harrison, Downey, Segal, and Howe (1971). Discriminative control was very rapidly achieved when the source of the sound stimulus and the response manipulandum were adjacent. According to Harrison 
et al. (1971), the sound orients the animal's head and eyes to the speaker source. "When the speaker and manipulandum are adjacent this results in the animal looking at the manipulandum. This biases the animal toward responding on that manipulandum (Harrison et al. 1971, Pp. 385)." The rapidity of acquisition of localized auditory control suggests that rapid autoshaping might be possible when an auditory stimulus is repeatedly paired with food presentations.

\section{Method}

Subjects. Subjects were six naive adult squirrel monkeys (Saimiri sciureus) maintained at free feeding weights for the first 25 sessions of the experiment. For the remaining sessions daily food intake was reduced until body weight reached $80 \%$ of free feeding weight. Maintenance at this weight continued for the duration of the experiment.

Apparatus. The experimental Plexiglas chamber was $9 \times 10 \times 11$ in. $(22.9 \times 25.4 \times 27.9 \mathrm{~cm})$. On one wall a standard translucent Gerbrands pigeon key $1-1 / 8$ in. $(2.9 \mathrm{~cm})$ in diam was mounted and recessed $3 / 16$ in. $(.5 \mathrm{~cm})$. The key could be illuminated by a 2.8 -W white light. An $8-0 h m ~ 1-3 / 4$ in. $(4.4 \mathrm{~cm})$ Midland speaker was mounted $3 / 4$ in. $(1.9 \mathrm{~cm})$ behind the key and derived its input from a BRS-Foringer audio oscillator (AO-201) connected to an audio amplifier (AA-202).

On the lower left hand corner of the same wall as the key and speaker a small wire mesh foodcup extended into the chamber. The center of the foodcup was $5.5 \mathrm{in} .(14.0 \mathrm{~cm})$ to the left of, and 5.0 in. $(12.7 \mathrm{~cm})$ below the center of the key. Delivery of 120-mg food pellets into the cup was accompanied bya slight click of the feeder mechanism.

A dimmed overhead 6-W houselight mounted to the enclosing sound-attenuated chamber and white noise delivery by an overhead speaker were on throughout the session. The intensity of the white noise as measured $1-1 / 2$ in. $(3.8 \mathrm{~cm})$ from the key was $50 \mathrm{~dB}$.

Sessions were controlled by standard BRS-Foringer circuitry and monitored by closed circuit television.

Procedure. Two groups of monkeys were magazine trained and subsequently exposed to the autoshaping procedure as described in Experiment I. Unlike the first experiment, however, tone bursts of $.2-\mathrm{sec}$ duration repeated at a rate of 1.2 bursts per sec were produced during trials by the speaker mounted directly behind the key. The frequency of the tone was $3333 \mathrm{cps}$ with a rise time of $.14 \mathrm{msec}$ and the intensity, as measured $1-1 / 2$ $(3.8 \mathrm{~cm})$ from the key, was $70 \mathrm{~dB}$.

The two groups of monkeys differed only with respect to the order of exposure to key illumination conditions. Group I animals (Monkeys 340, 341, and 342) were first exposed to the autoshaping procedure with the key continuously illuminated throughout the session, i.e., during both tone-signaled trials and intertrial intervals. For Group II animals (343, 344, and 365), the key was never illuminated during initial autoshaping sessions.

Subsequently, while the tone continued to signal food presentation, the illumination conditions were reversed such that the keylight was turned off throughout the session for two of the monkeys in Group I (341 and 342) and continuously illuminated for all three monkeys in Group II.

Two monkeys from Group I (341 and 342) were then reexposed to autoshaping with continuous key illumination until a criterion of responding on $90 \%$ of trials for 5 consecutive days had been reached. The negative response-dependency procedure was then introduced. In the absence of a keypress response a pellet was delivered at the end of the 10 -sec tone-signaled trial. If a keypress response occurred, however, the trial terminated immediately without delivery of the pellet. The key remained continuously illuminated through the sessions.

\section{Results}

Autoshaping: Group I. In two of the three monkeys in Group I, the keypress response was successfully autoshaped with the key continuously illuminated. The third monkey (340) made no more than three responses to the key during trials on any single session. As shown in Figure 2, Monkeys 341 and 342 made their first trial keypress response during Session 5 and 1, respectively. Responding reached $90 \%$ of trials after about 40 sessions. Monkey 341 pressed the key with his forehead while 342 pressed with his nose. For both monkeys there was a relatively large number of intertrial interval (ITI) responses at the beginning of acquisition, but stimulus control was evident once the monkeys were responding consistently.

When the key light was subsequently turned off for 26 sessions, Monkey 342 continued to respond on $90 \%-100 \%$ of trials while keypressing was eliminated immediately for Monkey 341. Reexposure to autoshaping with continuous key illumination did not affect the level of performance of Monkey 342, who continued to perform at the criterion level. This procedure resulted in rapid reacquisition of the keypress response for Monkey 341, with only 12 sessions needed to reach criterion.

Autoshaping: Group II. None of the monkeys in Group II acquired a keypress response when exposed to authoshaping with the key never illuminated. Over the course of 35 sessions, they made a total of 2,17 , and 13 responses, respectively, only 0,2 , and 3 of which occurred during trials. In two of the animals, head movements in the direction of the sound source were observed during trials. This was most evident in Monkey 344 where, during trials, he would approach the foodcup, rise, and alternately move his head from the foodcup to the area of the speaker and key.

Subsequent exposure to autoshaping with the key continuously illuminated did not lead to the acquisition of a key-press response in any of the three monkeys. Over the course of 25 sessions a total of 0,18 , and 37 responses were made, respectively, only 0,1 , and 0 of which occurred during trials. In all three animals, head

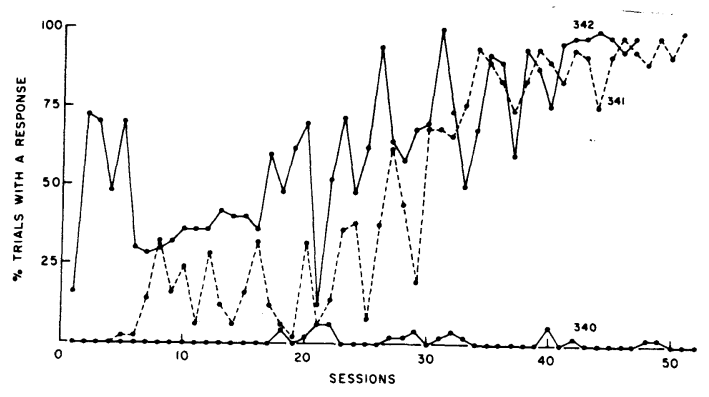

Figure 2. Acquisition of keypressing by autoshaping using an auditory signaling stimulus and continuous key illumination in three squirrel monkeys. Percent of daily trials to which there was a response. 
movements in the direction of the sound source were observed during trials.

Negative response dependency. This procedure resulted in the virtual elimination of keypress responding after 1-2 sessions. Results are shown in Figure 3 and are similar to that reported in Experiment I. Both monkeys were frequently observed, however, to make head movements in the direction of the sound source during trials.

\section{Discussion}

The results of this experiment make clear that the nature of the signaling stimulus is critical in the acquisition of autoshaped responding in the squirrel monkey. When food presentations were preceded by illumination of either an omnidirectional lever or key manipulandum (Gamzu \& Schwam, 1974), acquisition was about twice as rapid than when an auditory stimulus signaled food. Even then, the lack of responding in Group II animals with the key never illuminated indicated that repeated pairings of an auditory stimulus with food were not sufficient to generate autoshaped responding; rather, autoshaped responding was generated when initial exposure to such pairings was accompanied by continuous illumination of the key manipulandum. Illumination of the key may have increased the saliency of the key. As a consequence, the orienting responses elicited by the adjacent sound source might have been easily directed toward this area. Such an explanation might also account, in part, for the extremely rapid establishment of localized auditory stimulus control in squirrel monkeys reported by Harrison et al. (1971) since, in that study, the response manipulanda were continuously illuminated.

If this saliency hypothesis is correct, then Group II animals were initially exposed to a procedure similar to random food presentations. The subsequent failure of these animals to acquire the keypress response when the key was continuously illuminated may then parallel the interference with subsequent performance by random response-independent food presentations reported in squirrel monkeys (Gamzu \& Schwam, 1974) and pigeons (Gamzu \& Williams, 1971, 1973).

\section{CONCLUSIONS}

The elimination of autoshaped responding in the squirrel monkey reported here and elsewhere (Gamzu \& Schwam, 1974) cannot be attributed to the use of an inappropriate stimulus dimension to signal the presentation of food or an inappropriate response manipulandum, since the same negative response-dependency procedures resulted in similar effects. In effect, irrespective of stimulus dimension or response manipulandum, stimulus-food pairings were ineffective in maintaining responding when responding precluded the delivery of food. The relatively greater efficacy of visual as opposed to auditory stimuli in establishing autoshaped responding in the squirrel monkey is not surprising considering the "remarkably developed and advanced" visual system of the species (Rambaugh, 1968, Pp. 310). However, since the data pointed to differences between species, it was necessary to explore the experimental paradigm both with different manipulanda and different stimuli. Variability in the patterns of behavior just prior to consumption in the squirrel monkey may account for the behavioral differences found when this species is compared to pigeons in autoshaping procedures.

\section{REFERENCES}

Bubrey, J., \& Winokur, S. Controls for and constraints on auto thapling. Journal of the Experimental Analysis of Behavior, 1973, 20, 323-332.

Brown, P., Jenkins, H. M. Autoshaping of the pigeon's key

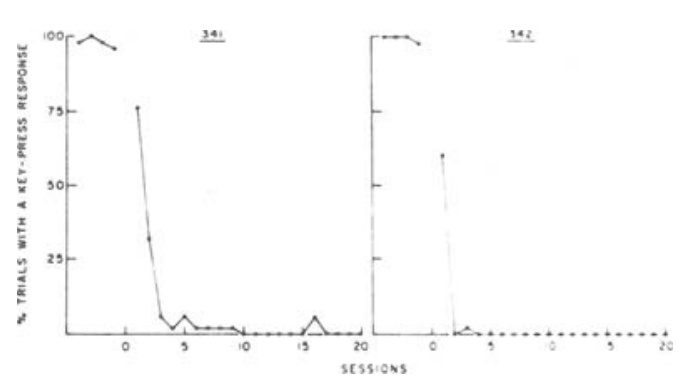

Figure 3. Percent of daily trials to which there was a keypress response during the negative response-dependency procedure. The upper left-half corner indicates responding during the preceding four autoshaping sessions.

peck. Journal of the Experimental Analysis of Beliavur, 1908. $11,1-8$.

Farthing. G. W. Effect of a signal previously paired with free food on operant response rate in pigeons. Psychonumic Science, $1971,23,343-344$.

Gamzu, E.. \& Schwam, E. Autoshaping and automaintenance of a key-press response in squirrel monkeys. Journal of tili Experimental Analysis of Behavior, 1974, 21, 361-371.

Gamzu, E., \& Williams, D. R. Classical conditioning of a complex skeletal response. Science, 1971, 171,923-925.

Gamzu, E. R., \& Williams, D. R. Associative factors underly in: the pigeon's key pecking in autoshaping procedure's. Juurnal of the experimental Analysis of Benavior, 1973, 19,2:25-232.

Harrison, J. M., Downey, P.. Segal, M. \& Howe, M. Control of responding by location of auditory stimuli: RApid acquisition in monkey and rat. Journal of the Experimental Analy sis of Behavior, $1971,15,379-386$

Jenkins, H. M., Moore, B. R. The form of the auto-shaped resuonse with food or water reinforcers. Journal of the Experimental Analysis of Behavior, 1973, 20, 163-181.

Moore, $B$. R. The role of directed Pavlovian reactions in simple instrumental learning in the pigeon. In $R$. A. Hinde \& $J$. Stevenson-Hinde (Eds.), Constraints on learning. New York: Academic Press, 1973, 159-188.

Peterson, G. B., Ackil, J. E., Frommer, G. P. \& Hearst, E. S. Conditioned approach and contact behavior toward signals for food or brain-stimulation reinforcement. Science, 1972, 177. 1009-1011.

Riddle, W. C., Rednick, A. B., Catania, A. C., \& Tucker, S. J.

Riddle, W. C., Rednick, A. B.. Catania, A. C., \& Tucker, S. J.
Complete squirrel monkeys diet in tablet form. Journal of the Experimental Analy sis of Behavior, 1966,9,670.

Rumbaugh, D. The learning and sensory capacities of the squirrel monkey in phylogenetic perspective. In L. A. Rosenblum and R. W. Cooper (Eds.), The squirrel monkey. New York: Acadenic Press, 1968.

Schwartz, B., \& Williams D. R. Two different kinds of key p :ck in the pigeon: some properties of responses muintained by negative and positive response-reinforcer contingencies. Journal of the Experimental Analy sis of Behavior, 1972a, 18 , $201 \cdot 216$

Schwart $\%$, B., \& Williams, D. R. The role of the response-reiniorcer contingency in negative untomaintenance. Journal of the Experimental Analy sis of Behavior, 19721, 17. $351-357$.

Squier, L. H. Autoshaping key responses with fish. Psychonomic Science, $1969,17,177-178$.

Wasserman, E. A. The effect of redundant contextual stimuil on autoshaping the pigeon's keypeck. Animal Learning \& Behavior, 1973, 1, 198-206.

Williams, U. R., \& Williams, H. Automaintenance in the pigeon: sustained pecking despite contingent nonreinforcement. Journal of the Experimental Analysis of Behavior, 1969, 12 , 511-520.

Ziegler, H. Feeding behavior in the pigeon: a neurobehavioral analysis. In I. Goodman and M. Schein (Eds.), Birds: Brain and behavior. New York: Academic Press, in press.

\section{REFERENCE NOTE}

H. Zeigler and P. Welch, The "consummatory" response of feeding in the pigeon: temporal organization and neurosensory control. Paper presented at the meeting of the American Psychological Association, Honolulu, Hawaii, 1972.

(Received for publication December 30, 1974.) 